\section{Combining Endoscopy with DICIDA Scanning to Evaluate Prolonged Epigastralgia After Choledochoduodenostomy}

Side-to-side choledochoduodenostomy is widely accepted as a safe and effective therapeutic maneuver conditions, such as cholelithiasis, distal biliary stricture, stenosis of the sphincter of Oddi, chronic biliary pancreatitis, duodenal diverticulum, and other biliary obstructions, whether benign or malignant (1).

The short-term mortality is low, ranging from $1.4 \%$ to $3.5 \%$, and the most severe complication, anastomotic leakage, occurs in only $2-5 \%$ (1). With regard to long-term outcome, retrograde cholangitis and sump syndrome are the two major complications. Despite some opinions the contrary, such complications are only sporadically observed, with an incidence of $1.6-3.3 \%(2,3)$. Less common complications are retained stones, stitch ulcer, and nonspecific gastric irritation caused by bile reflux. Routine endoscopic examination offers a convenient way of differentiating between these complications, allowing checking of the stoma patency and the blind sump. In addition, the large stoma offers an adequate lumen for the endoscopic removal of retained stones; and endoscopic sphincterotomy is the therapy of choice for the sump syndrome (4). However, monitoring the dynamic motility of bile flow after anatomic alterations cannot be carried out by endoscopy alone. The DICIDA scan, a radioactive isotope study, makes available a definitive diagnostic approach to the biliary system (5). It not only discloses the patency of the biliary system and stoma, but also sensitively records enterogastric reflux using a time-sequence exposure. Combining endoscopy with the DICIDA scan, postoperative long-term follow-up can be made more precise.
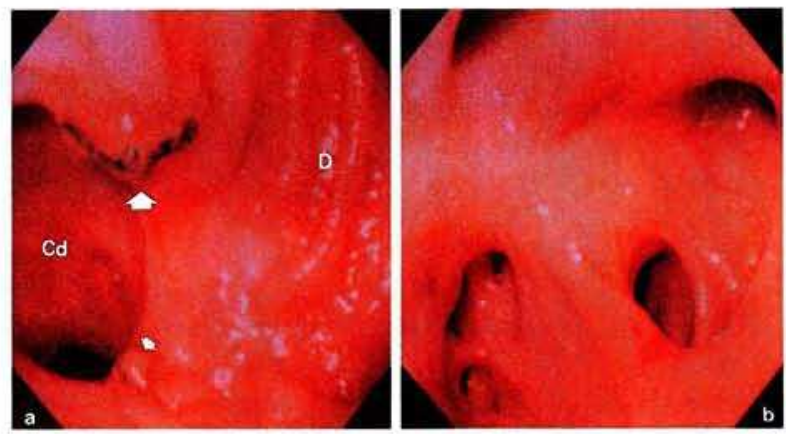

Figure 1 a: The stoma area of the choledochoduodenostomy shows a retained stitch with granuloma (arrows). The choledochal (Cd) and duodenal (D) orifiices were both patent. b The choledochal part was patent, without any food debris.
We report here on a case of a 44-year-old male who had undergone cholecystectomy 22 years previously. He was suffering from common bile duct and intrahepatic stones, with suppurative cholangitis. The common bile duct was dilated to $2.0 \mathrm{~cm}$ in size. Choledocholithotomy and side-to-side choledochoduodenostomy were performed without complications. No biliary tract infection or retained stones were found during follow-up. However, the patient experienced prolonged epigastralgia for eight months after the operation. Panendoscopy showed that the stoma was patent, except for a retained stitch with granuloma formation over the stoma suture (Figure 1a). The sump syndrome was excluded, as there was no food or retained stone in the biliary system (Figure $\mathbf{1 b}$ ). The stitch was removed endoscopically, and $\mathrm{H}_{2}$-blockers were administered, leading to ulcer healing within two months. The clinical complain improved considerably, but indolent postprandial fullness persisted. Significant enterogastric reflux was confirmed by the presence of retrograde radioactive staining in the stomach, 45 minutes after starting a DICIDA scan (Figure 2). As there was quick fading of the stain within 120 minutes, the patient's reflux was not so prominent as to cause severe complications.

Enterogastric reflux is likely to cause gastric upset in patients who have undergone choledochoduodenostomy. It would therefore be of interest to see whether the severity of the reflux could be assessed using DICIDA scanning and whether the scan might correlate with pathologic findings in the gastric mucosa after choledochoduodenostomy. We believe that combining endoscopy with DICIDA scanning may provide an excellent approach to the long-term follow-up after choledochoduodenostomy.

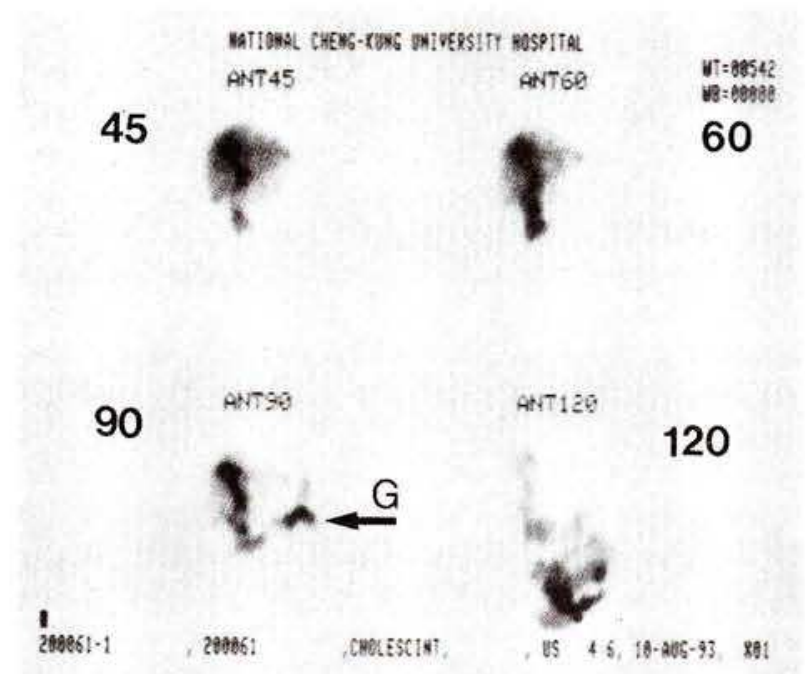

Figure 2: The radioactive isotope $(G)$ was retained in the stomach (arrow) 90 minutes after starting the DICIDA scan, indicating retrograde enterogastric reflux. However, it faded out at 120 minutes. 


\section{References}

1. De La Cuadra R, Csendes A, Braghetto I, et al.: Indications and results of choledochoduodenostomy in benign biliary tract disease. Hepatogastroenterol 1989; 36: 147

2. Baker AR, Neoptolemos JP, Leese T, Fossard DP: Choledochoduodenostomy, transduodenal sphincteroplasty and sphincterotomy for calculi of the common bile duct. Surg Gynecol Obstet 1987; 164: 245.

3. Cubillos L, Fiallo R, Rodriguez J: Is choledochoduodenostomy in the treatment of stones in the common bile duct an obsolete technique? World J Surg 1985; 9: 484.

4. Baker AR, Neoptolemon JP, Carr-Locke DL, Fossard DP: Sump syndrome following choledochoduodenostomy and its endoscopic treatment. Br J Surg 1985; 72: 433-435.

5. Papadimitriou J, Fotopoulos A, Tsiftsis D, et al.: Dynamic evaluation of the long-term patency of choledochoduodenostomy compared with sphincteroplasty. Surg Gynecol Obstet 1982; 155: 17.



${ }^{1}$ Department of Internal Medicine

${ }^{2}$ Department of General Surgery, National Cheng Kung University Hospital, Tainan, Taiwan

Corresponding Author

P.-W. Lin, M.D.

Department of General Surgery

National Cheng Kung University Hospital

138 Sheng Li Road

Tainan 70428

Taiwan

Fax: +886-6-2370941 